\title{
ERK1/2 pathway mediates epithelial-mesenchymal transition by cross-interacting with TGF//Smad and Jagged/Notch signaling pathways in lens epithelial cells
}

\author{
XIAOYUN CHEN*, SHAOBI YE* , WEI XIAO, WENCONG WANG, LIXIA LUO and YIZHI LIU \\ State Key Laboratory of Ophthalmology, Zhongshan Ophthalmic Center, \\ Sun Yat-sen University, Guangzhou, Guangdong 510060, P.R. China \\ Received February 6, 2014; Accepted April 2, 2014
}

DOI: $10.3892 /$ ijmm.2014.1723

\begin{abstract}
Epithelial-mesenchymal transition (EMT) of lens epithelial cells (LECs) is the major pathological mechanism in anterior subcapsular cataract (ASC) and posterior capsule opacification (PCO), which are important causes of visual impairment. Extracellular signal-regulated kinase (ERK)1/2 pathway has been reported to play a major role in carcinogenesis, cancer metastasis and various fibrotic diseases. We hypothesized that ERK1/2 signaling can cross-interact with canonical transforming growth factor $\beta$ (TGF $\beta$ )/Smad signaling and the Notch pathway, which subsequently contributes to LECs EMT. In this study, we demonstrated that ERK1/2 signaling was activated in TGF $\beta 2$-induced EMT in human LECs, whereas the blockade of TGF $\beta 2 / \mathrm{Smad} 2 / 3$ signaling with SB431542 did not inhibit the activation of ERK1/2 induced by TGF $\beta 2$. In addition, inactivation of ERK1/2 signaling with a specific MEK/ ERK1/2 inhibitor, U0126, completely prevented the TGF $\beta 2$ induced upregulation of $\alpha$-SMA, collagen type I, collagen type IV and fibronectin. We also demonstrated that inactivation of ERK1/2 signaling inhibited canonical TGF $\beta / \mathrm{Smad}$ signaling, as well as the Jagged/Notch pathway. By contrast, blockade of the Notch pathway by DAPT inhibited the TGF $\beta 2$-induced activation of ERK1/2 pathway in LECs. Thus, results of this study provide evidence for the complex interplay between ERK1/2, TGF $\beta /$ Smad, and Jagged/Notch signaling pathways in the regulation of EMT in LECs. Inhibition of the
\end{abstract}

Correspondence to: Professor Yizhi Liu, State Key Laboratory of Ophthalmology, Zhongshan Ophthalmic Center, Sun Yat-sen University, 54 Xianlie Road, Guangzhou, Guangdong 510060, P.R. China

E-mail: yizhi_liu@aliyun.com

*Contributed equally

Key words: extracellular signal-regulated kinase1/2 pathway, epithelial-mesenchymal transition, lens epithelial cells, Notch pathway, anterior subcapsular cataract, posterior capsule opacification
ERK1/2 pathway may therefore have therapeutic value in the prevention and treatment of ASC and PCO.

\section{Introduction}

Cataract is the most common cause of visual impairment in the elderly worldwide, particularly in developing countries (1). Anterior subcapsular cataract (ASC) and posterior capsule opacification (PCO) are different types of cataract that share similar cellular and molecular features $(2,3)$. PCO, also known as a secondary cataract, is the most common long-term complication of modern cataract surgery. In the past few decades, although advances in surgical techniques, intraocular lens materials and designs have reduced the PCO rate, the incidence of PCO is still $20-40 \%$ in adults and $100 \%$ in children $(4,5)$. At present, cataract surgery and Nd:YAG laser capsulotomy are the only effective treatments for ASC and PCO, however, they are likely to induce many other complications and risks. Therefore, a better understanding of the pathogenesis of these diseases is critical for the development of new pharmacologic treatments.

Accumulating evidence has shown that the epithelialmesenchymal transition (EMT) of lens epithelial cells (LECs) is a key pathological mechanism involved in the development of ASC $(6,7)$ and PCO $(8,9)$. PCO is caused by a wound healing response of residual LECs following cataract surgery. After surgery, the levels of various cytokines and growth factors increase in the aqueous humor and stimulate the residual LECs to proliferate and undergo EMT (10). Transforming growth factor $\beta$ (TGF $\beta$ ), especially TGF $\beta 2$, the major isoform in the aqueous humor of the eye, plays a central role in the cell biology of PCO (11). During the process of EMT, LECs undergo cytoskeletal rearrangement and loss of epithelial phenotype, then migrate away from the original location onto the posterior capsule, with the addition of a large amount of extracellular matrix proteins (collagen and fibronectin) deposition, and finally contribute to the development of PCO $(7,10)$. Unlike $\mathrm{PCO}, \mathrm{ASC}$ is a primary cataract that is mainly caused by ocular trauma, inflammation or irritation (12). The proliferation and EMT of LECs in situ lead to the formation of subcapsular plaques just beneath the lens anterior capsule, similar to the transdifferentiated cells in PCO (2). Thus, inhibition of the 
proliferation of LECs and EMT may be a promising strategy to prevent ASC and PCO.

Several signaling pathways are involved in the process of LECs EMT in ASC and PCO development. Among these, canonical TGF $\beta /$ Smad signaling has been identified to occupy a crucial position in the signaling networks that control EMT of LECs. TGF $\beta /$ Smad signaling transmits signals by binding to the related transmembrane type I and II receptors, which subsequently phosphorylate receptor-regulated Smad2 and Smad3 (13). The phosphorylated Smad2/3 bind to the common mediator Smad4 to form a stable hetero-oligomeric complex, and then the complex translocates to the nucleus where the target gene expression is regulated (13). Recent studies have demonstrated that the blockade of TGF $\beta 2 / \mathrm{Smad} 2 / 3$ efficiently prevents the effect of TGF $\beta 2$ on LECs migration, extracellular matrix production and EMT $(14,15)$. In addition to the canonical Smad signaling, extracellular signal-regulated kinase (ERK) signaling is involved in TGF $\beta$-induced EMT in different types of cells (16-19). The activation of ERK1/2 signaling enhances TGF $\beta$-induced EMT, accompanied by morphological changes, the upregulation of EMT markers and extracellular matrix components. Blocking the function of ERK1/2 using a special inhibitor results in the inhibition of TGF $\beta$-induced EMT (17,20). In LECs EMT, it has been previously reported that ERK1/2 is rapidly activated by TGF $\beta$, and the specific inhibitor of ERK1/2 blocks the morphologic change of LECs and the upregulation of Slug induced by TGF $\beta$ (19).

Although the role of ERK1/2 signaling in EMT during cancer progression and some fibrotic disorders has been studied, the interaction of ERK1/2 with the canonical TGF $\beta /$ Smad signaling pathway and other signaling pathways in fibrotic diseases is poorly understood. In this study, we demonstrated that the TGF $\beta 2$-induced activation of ERK $1 / 2$ is independent of $\mathrm{TGF} \beta / \mathrm{Smad}$ signaling in human LECs, while the blockade of ERK1/2 signaling with the inhibitor U0126 completely prevents TGF 32 -induced EMT. Moreover, blockade of ERK1/2 signaling inhibits the canonical Smad signaling pathway, as well as the Jagged/Notch pathway. We also found that non-canonical TGF $\beta / E R K 1 / 2$ signaling can also be mediated by the Notch pathway. Taken together, these results suggested that ERK1/2 signaling cross-interacts with the TGF $\beta /$ Smad and the Jagged/Notch signaling pathways, thus mediating EMT in LECs.

\section{Materials and methods}

Reagents and antibodies. U0126 (a selective inhibitor of MEK1 and MEK2) and recombinant human TGF $\beta 2$ were purchased from Cell Signaling Technology, Inc. (Danvers, MA, USA). SB431542 (a specific inhibitor for TGF $\beta$ receptor type I/ALK5 kinase that phosphorylates Smad2/3) and DAPT (an inhibitor of Notch receptor cleavage) were purchased from Sigma-Aldrich (St. Louis, MO, USA). Antibodies against ERK1/2, p-ERK1/2, Jagged-1, Notch-1, Notch-2, p-Smad2, p-Smad3, goat anti-rabbit and horse anti-mouse horseradish peroxidase (HRP)-conjugated secondary antibodies were purchased from Cell Signaling Technology Inc. Antibodies against $\beta$-actin, $\alpha$-SMA, collagen type I (Col I), collagen type IV (Col IV), and fibronectin (FN) were purchased from Abcam (Cambridge, UK).
Cell culture and treatment. The SRA01/04 human LEC line was kindly provided by Professor Fu Shang at the Laboratory for Nutrition and Vision Research (Boston, MA, USA), and cultured in Dulbecco's modified Eagle's medium (DMEM) containing $10 \%$ fetal bovine serum (FBS). The cells were grown at $37^{\circ} \mathrm{C}$ in a humidified atmosphere containing $5 \% \mathrm{CO}_{2}$ and dissociated with $0.25 \%$ trypsin- $0.02 \%$ ethylenediaminetetraacetic acid (EDTA) solution.

For TGF 32 and U0126 treatments, the cells were seeded in 6-well plates and treated with $10 \mathrm{ng} / \mathrm{ml}$ recombinant human TGF $\beta 2$ and different concentrations of U0126 for different time-points.

Quantitative PCR analysis for gene expression. Total RNA was isolated from LECs using TRIzol reagent (Invitrogen, Carlsbad, CA, USA), and the RNA was then treated with DNase I (Sigma-Aldrich) to remove genomic DNA contamination. The concentration of total RNA was quantified by spectrophotometry and cDNA was synthesized with a reverse transcription kit (Takara Bio Inc., Otsu, Japan). For quantitative analysis of mRNA expression, the SYBR PrimeScript RT-PCR kit (Takara Bio Inc.) was used to amplify the target genes, and the reactions were performed with the ABI Prism 7000 sequence detection system (Applied Biosystems, Foster City, CA, USA) according to the manufacturer's protocol. Glyceraldehyde 3-phosphate dehydrogenase (GAPDH) was used as an internal control.

Western blot analysis for protein expression. The cells were washed twice with PBS, and then lysed in $100 \mu 1$ of RIPA buffer with protease inhibitor cocktail for total protein extraction. Protein was collected after centrifugation and mixed with 5X SDS sample buffer. The samples were separated by $10 \%$ SDS-PAGE, and then transferred to PVDF membranes. The membranes were blocked with $5 \%$ non-fat milk for $1 \mathrm{~h}$ and the membranes were subsequently incubated with different primary antibodies at $4^{\circ} \mathrm{C}$ overnight. The membranes were washed with $1 \mathrm{X}$ PBS containing $0.1 \%$ Tween-20 (PBST) three times, and incubated with HRP-conjugated secondary antibodies for $1 \mathrm{~h}$ at room temperature. The protein bands were detected with chemiluminescence detection reagents. $\beta$-actin was used as the loading control. Densitometric analysis was conducted by ImageJ software 1.41 (National Institutes of Health, Bethesda, MD, USA).

Statistical analysis. Experiments presented in the figures are representative of three or more different repetitions. Data were presented as mean \pm standard error of the mean (SEM) and analyzed with SPSS 15.0 software (SPSS, Inc., Chicago, IL, USA). A standard Student's t-test was used for statistical analysis. $\mathrm{P}<0.05$ was considered to indicate statistical significance.

\section{Results}

Blockade of ERK1/2 signaling by U0126 prevents TGF $\beta 2$ induced EMT in LECs. To examine whether the blockade of ERK1/2 signaling prevented TGF 32 -induced EMT in LECs, U0126 (a selective inhibitor of MEK1 and MEK2) was used. EMT markers such as $\alpha$-SMA, Col I, Col IV and FN 

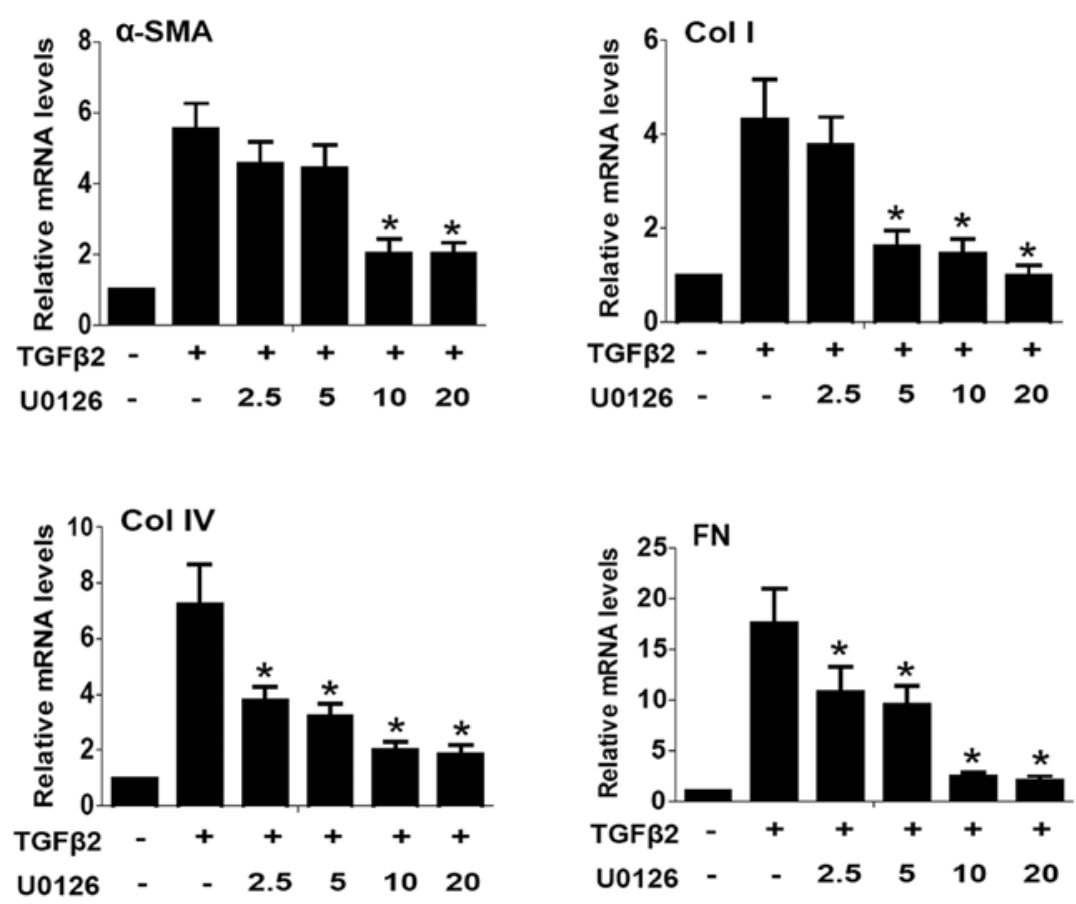

Figure 1. Blockade of extracellular signal-regulated kinase (ERK)1/2 pathway by U0126 downregulates epithelial-mesenchymal transition (EMT) markers mRNA expression induced by transforming growth factor $\beta$ (TGF $\beta) 2$. Lens epithelial cells (LECs) were cultured in the absence or presence of TGF $\beta 2$ (10 ng/ml) with U0126 (2.5, 5.0, 10.0 and $20.0 \mu \mathrm{M})$ or DMSO for $24 \mathrm{~h}$. The mRNA expression levels of $\alpha$-SMA, collagen type I (Col I), collagen type IV (Col IV) and fibronectin (FN) were determined by quantitative PCR. Gene expression levels were normalized to the GAPDH control. "P<0.05 vs. TGF $\beta 2$ treated with DMSO group.

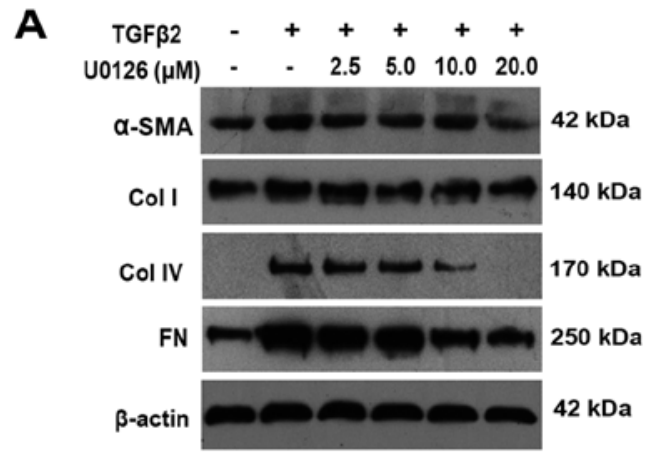

B

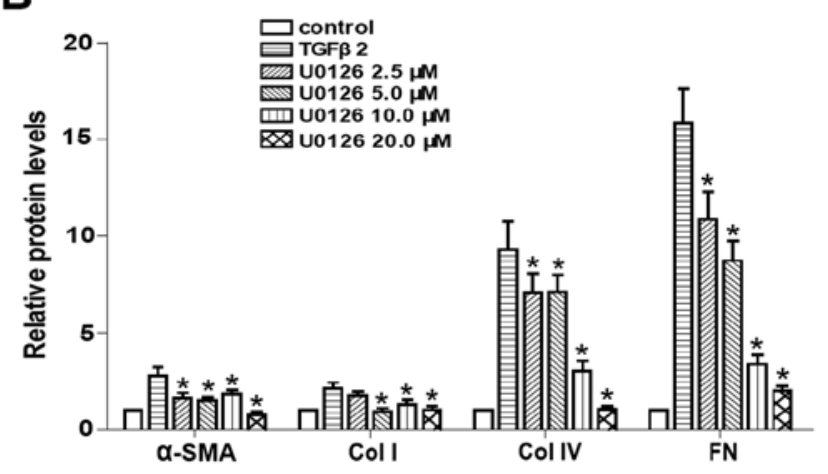

Figure 2. U0126 downregulates epithelial-mesenchymal transition (EMT) markers protein expression induced by transforming grow th factor $\beta$ (TGF $\beta) 2$. Lens epithelial cells (LECs) were cultured in the absence or presence of TGF $\beta 2(10 \mathrm{ng} / \mathrm{ml})$ with U0126 $(2.5,5.0,10.0$ and $20.0 \mu \mathrm{M})$ or DMSO for 24 h. (A) The protein expression levels of $\alpha$-SMA, collagen type I (Col I), collagen type IV (Col IV) and fibronectin (FN) were detected by western blot analysis. (B) Quantification of protein levels from three independent experiments. ${ }^{*} \mathrm{P}<0.05$ vs. TGF $\beta 2$ treated with DMSO group. were investigated at mRNA and protein levels by quantitative PCR and western blot analysis, respectively. As shown in Fig. 1, quantitative PCR results showed that the mRNA expression of $\alpha$-SMA, Co1 I, Col IV and FN were upregulated 5.5- 4.3- 7.2- and 17.7-fold in TGF 32 -induced LECs for $24 \mathrm{~h}$. In addition, western blot analysis results showed that TGF 32 significantly increased the protein expression of $\alpha$-SMA, Col I, Col IV and FN in LECs (Fig. 2). Co-treatment with U0126 markedly abrogated the upregulation of $\alpha$-SMA, Col I, Col IV and FN induced by TGF 32 at the mRNA and protein levels (Figs. 1 and 2: P<0.05 vs. TGF $\beta 2$ treated with DMSO group). Maximum effect of U0126 was observed at a concentration of $20.0 \mu \mathrm{M}$, however, there was no obvious difference between 10.0 and $20.0 \mu \mathrm{M}$ at mRNA level. These data suggested that the blockade of ERK1/2 pathway by U0126 effectively attenuated TGF $\beta 2$-induced EMT in LECs.

TGF $\beta 2$-induced ERK1/2 activation is independent of the canonical TGF $/$ Smad pathway. To determine whether the canonical TGF $\beta /$ Smad signaling is required for the activation of ERK1/2 pathway by TGF 32 , SB431542 (a specific inhibitor for TGF $\beta$ receptor type I/ALK5 kinase that phosphorylates Smad2/3) was used. As shown in Fig. 3A and B, when LECs were stimulated by TGF $\beta 2$ for 30 min, ERK1/2 was activated via phosphorylation but with an unchanged total protein level, while U0126 treatment completely inhibited the TGF $\beta 2$ induced activation of ERK1/2. However, SB431542 treatment had no effect on the phosphorylation of ERK1/2 (Fig. 3A and B: $\mathrm{P}<0.05$ vs. TGF $\beta 2$ treated with DMSO group). These results indicated that TGF 32 -induced ERK1/2 activation is independent of the canonical TGF $\beta /$ Smad pathway in LECs. 


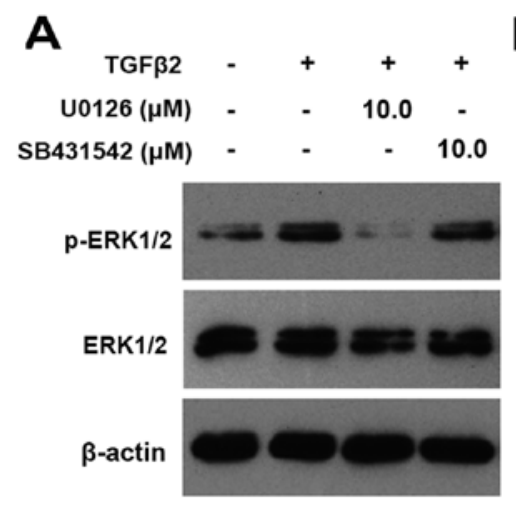

B
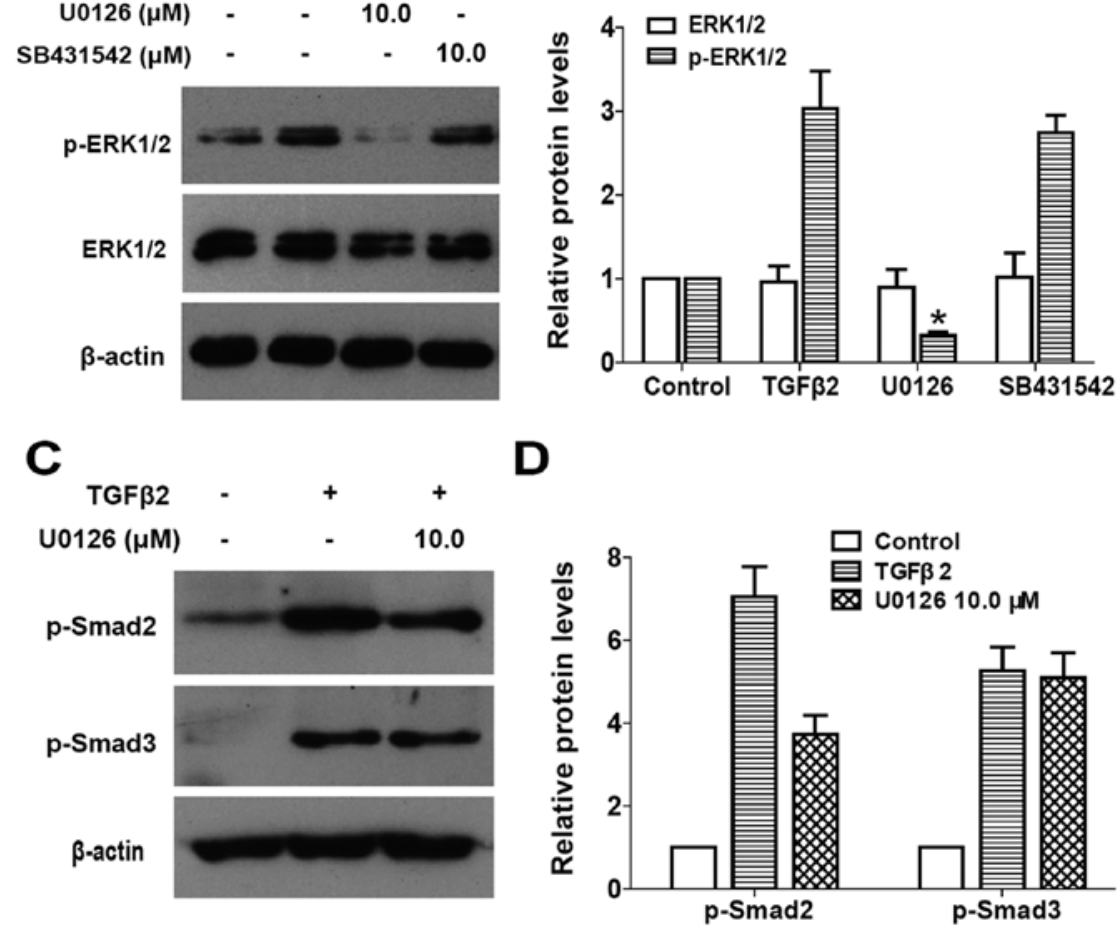

Figure 3. Transforming growth factor $\beta$ (TGF $\beta$ )2-induced extracellular signal-regulated kinase (ERK) $1 / 2$ activation is independent of TGF $\beta /$ Smad signaling and U0126 inhibits TGF 32 -induced phosphorylation of Smad2. (A) Lens epithelial cells (LECs) were cultured in the absence or presence of TGF 32 with $\mathrm{U} 0126(10.0 \mu \mathrm{M})$ or SB431542 $(10.0 \mu \mathrm{M})$ for $30 \mathrm{~min}$, while the expression levels of p-ERK1/2 and ERK1/2 were determined by western blot analysis. (B) Quantification of protein levels from three independent experiments. "P $<0.05$ vs. TGF $\beta 2$ treated with DMSO group. (C) The phosphorylation levels of Smad2 and Smad3 were detected by western blot analysis after 60-min treatment. (D) Quantification of protein levels from three independent experiments. ${ }^{*} \mathrm{P}<0.05$ vs. TGF 32 treated with DMSO group.

U0126 mediates canonical TGF $\beta /$ Smad signaling by inhibiting the phosphorylation of Smad2. To examine whether there is a crosstalk between the ERK1/2 signaling and the canonical TGF $\beta /$ Smad pathway, the effect of U0126 on the activation of receptor-regulated Smad proteins Smad2 and Smad3 was examined. As shown in Fig. 3C and D, TGF $\beta 2$ alone clearly induced apparent phosphorylation of Smad2 and Smad3 following 60-min treatment, whereas co-treatment with U0126 inhibited the phosphorylation of Smad2, but had no effect on the phosphorylation of Smad3 in LECs (Fig. 3C and D: $\mathrm{P}<0.05$ vs. TGF $\beta 2$ treated with DMSO group). Collectively, these data suggested that U0126 inhibits the canonical TGF $32 /$ Smad signaling transduction by inhibiting the phosphorylation of Smad2. Thus, there is a crosstalk between ERK1/2 signaling and the canonical TGF $32 /$ Smad signaling pathway in LECs.

U0126 prevents TGF 32 -induced EMT partly by inhibiting the Jagged/Notch pathway. Accumulating evidence suggests that the Notch signaling pathway is a vital regulator in the induction of EMT during embryonic development, cancer metastasis and various fibrotic diseases (21). Results of a previous study also found that the Jagged/Notch pathway is activated through canonical TGF $\beta 2 /$ Smad signaling during EMT in human LECs, while blockade of the Notch pathway with the specific inhibitor DAPT strongly inhibited TGF 32 induced EMT (unpublished data). Therefore, we investigated whether inactivation of ERK1/2 signaling with U0126 inhibited Notch signaling activated by TGF $\beta 2$, and subsequently inhibited LECs EMT. As shown in Figs. 4 and 5, TGF $\beta 2$ treatment alone significantly increased the expression of Jagged-1, Notch-1 and Notch-2 at mRNA and protein levels, while U0126 completely attenuated the TGF 32 -induced upregulation of Jagged-1, Notch-1 and Notch-2 (Figs. 4 and 5: $\mathrm{P}<0.05$ vs. TGF $\beta 2$ treated with DMSO group). In addition, U0126 treatment attenuated TGF $\beta 2$-induced Notch target genes Hes-1 and Hey-1 expression (Fig. 4: $\mathrm{P}<0.05$ vs. TGF $\beta 2$ treated with DMSO group). These results suggested that U0126 prevents TGF $\beta 2$-induced EMT partly by downregulating the Jagged/Notch pathway. Thus, non-canonical ERK1/2 signaling also contributes to the TGF $\beta 2$-induced activation of the Notch pathway in LECs.

Non-canonical TGF $/$ ERK1/2 signaling can be mediated by the Notch pathway. It is unclear whether blockade of Notch signaling is able to modulate ERK1/2 signaling pathway activated by TGF $\beta 2$. As expected, blockade of the Notch pathway by DAPT clearly inhibited the TGF 32 -induced activation of ERK1/2 pathway in LECs (Fig. 6: $\mathrm{P}<0.05$ vs. TGF $\beta 2$ treated with DMSO group). These results suggested that the non-canonical TGF $\beta /$ ERK1/2 signaling can be mediated by the Notch pathway conversely in LECs. This finding also indicated that there is a crosstalk between the ERK1/2 signaling and Notch pathways. 

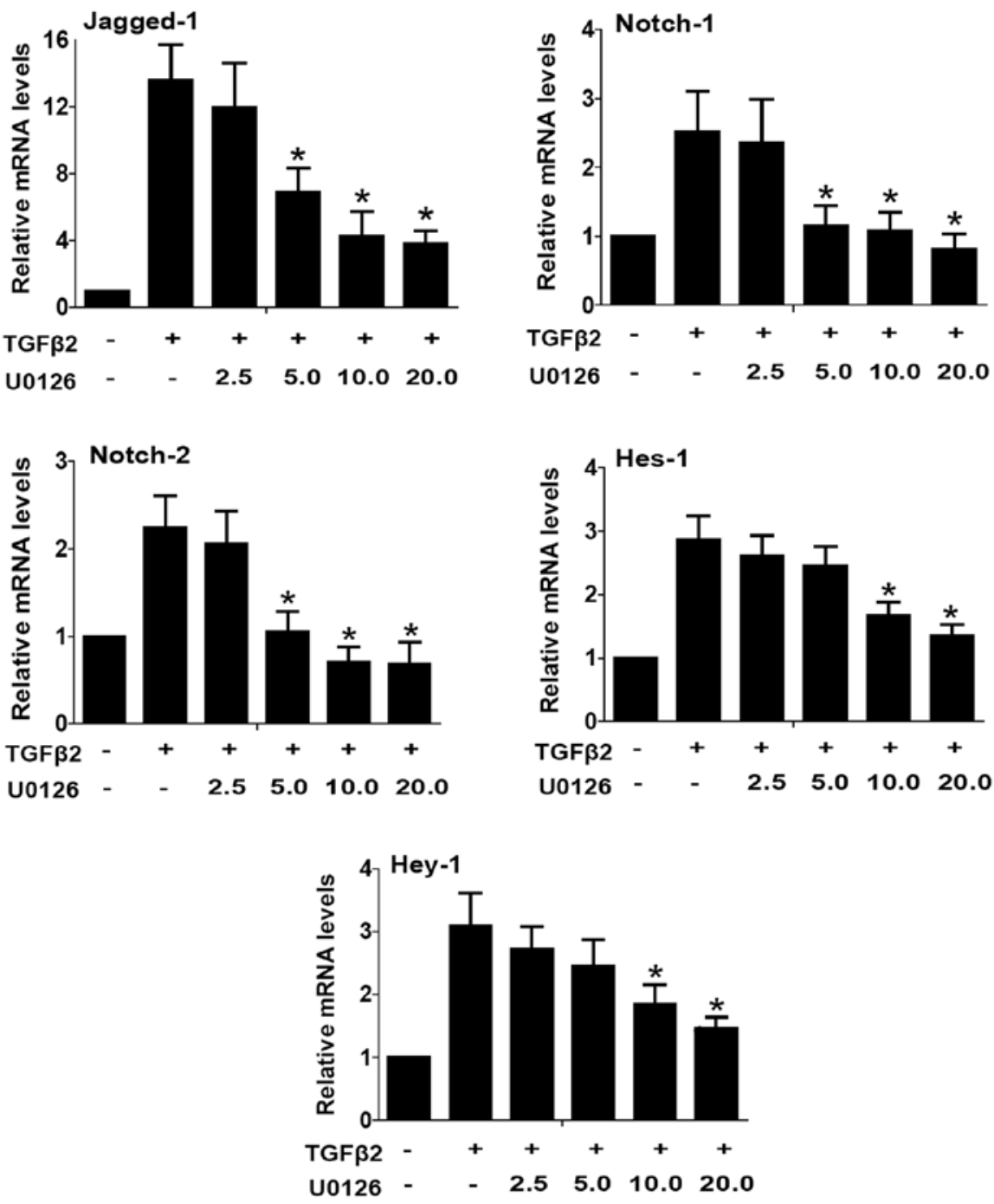

Figure 4. U0126 prevents transforming growth factor $\beta$ (TGF $\beta$ )2-induced epithelial-mesenchymal transition (EMT) by downregulating the Notch pathwayrelated genes mRNA expression. Lens epithelial cells (LECs) were treated with TGF 32 in the presence of U0126 (2.5, 5.0, 10.0 and $20.0 \mu \mathrm{M})$ or DMSO for $24 \mathrm{~h}$, while the mRNA expression levels of Jagged-1, Notch-1, Notch-2, Hes-1 and Hey-1 were detected by quantitative PCR. Gene levels were normalized to the control GAPDH. ${ }^{*} \mathrm{P}<0.05$ vs. TGF $\beta 2$ treated with DMSO group.

\section{Discussion}

A growing number of studies have proven that the development of ASC and PCO largely attributes to the EMT of LECs in response to a variety of cytokines, typically TGF $\beta 2$. Activation of ERK1/2 pathway plays a critical role in carcinogenesis, cancer metastasis, and various fibrotic diseases, including PCO (19,22-24). In this study, we investigated the role of ERK1/2 signaling in TGF 32 -induced EMT in human LECs, with a focus on the interaction of ERK1/2 signaling with the canonical TGF $32 /$ Smad and the Jagged/Notch pathways. We found that the activation of ERK1/2 signaling by TGF $\beta 2$ is independent of canonical TGF $32 /$ Smad signaling in LECs, while the blockade of ERK1/2 signaling with U0126 markedly prevented TGF 32 -induced EMT. Furthermore, the blockade of ERK1/2 signaling inhibits the canonical Smad signaling pathway, as well as the Jagged/Notch pathway. By contrast, we demonstrated that non-canonical TGF $\beta /$ ERK1/2 signaling can also be mediated by the Notch pathway. Therefore, our data suggest that ERK1/2 signaling cross-interacts with the canonical TGF $\beta /$ Smad and Jagged/Notch signaling pathways, thus regulating EMT in LECs.

TGF $\beta$ signaling occupies a key position in the signaling networks that regulates EMT. It includes canonical Smad signaling and non-canonical Smad independent signaling pathways. Previous studies have reported that ERK1/2 signaling is involved in TGF $\beta$-induced EMT in LECs and other types of cells (16-19). The activation of ERK1/2 signaling promotes TGF $\beta$-induced EMT and ECM components deposition, whereas the inactivation of ERK1/2 inhibits TGF $\beta$-induced EMT effectively $(19,20)$. In the present study, we found that ERK1/2 is rapidly activated by TGF 32 stimulation, and that MEK1/2 inhibitor U0126 blocks this response completely. Nevertheless, SB431542, a specific inhibitor for the canonical $\mathrm{TGF} \beta / \mathrm{Smad} 2 / 3$ signaling transduction, has no effect on the activation of ERK $1 / 2$ induced by TGF $\beta 2$. These data indicate that TGF $\beta 2$-induced ERK1/2 activation is independent of the TGF $\beta /$ Smad pathway in LECs. In addition, inactivation of ERK1/2 signaling strongly prevents the upregulation of EMT markers induced by TGF $\beta 2$. These results suggest that ERK1/2 
A

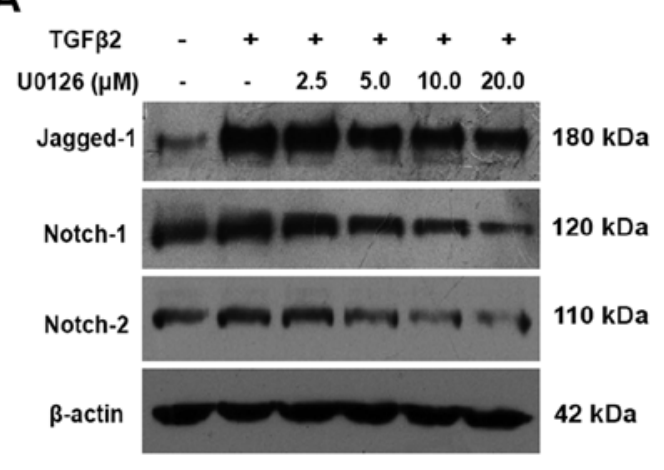

B

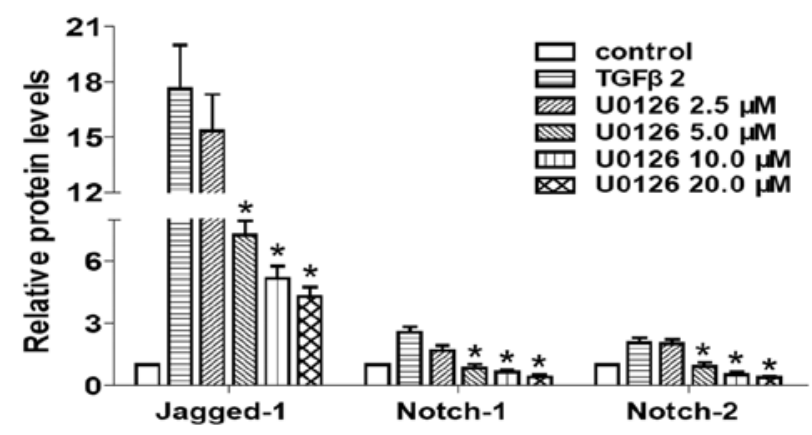

Figure 5. U0126 prevents transforming growth factor $\beta$ (TGF $\beta$ )2-induced epithelial-mesenchymal transition (EMT) by downregulating the Notch pathway-related genes protein expression. (A) Lens epithelial cells (LECs) were treated with TGF $\beta 2$ in the presence of $\operatorname{U0126}(2.5,5.0,10.0$ and $20.0 \mu \mathrm{M})$ or DMSO for $24 \mathrm{~h}$, the protein expression levels of Jagged-1, Notch-1 and Notch-2 were detected by western blot analysis. (B) Quantification of protein levels from three independent experiments. ${ }^{*} \mathrm{P}<0.05$ vs. TGF $\beta 2$ treated with DMSO group.

signaling pathway is a critical mediator for TGF $\beta$ induction of EMT in LECs, and ERK1/2 inhibitor can be useful for abrogating EMT phenotype.

It has been reported that non-canonical Smad signaling, such as the p38MAPK and PI3K/AKT pathways, can crosstalk and integrate with the canonical TGF $\beta /$ Smad signaling, thereby contributing to EMT (25). To examine whether there is a crosstalk between the non-canonical TGF $\beta / E R K 1 / 2$ signaling and the canonical TGF $\beta / \mathrm{Smad}$ signaling, the effect of U0126 on the activation of receptor-regulated Smad2 and Smad3 induced by TGF $\beta 2$ was investigated. We found that U0126 inhibits the phosphorylation of Smad 2 induced by TGF $\beta 2$, but cannot inhibit the phosphorylation of Smad3 in LECs. These results suggest that U0126 mediates the canonical TGF $\beta /$ Smad signaling by inhibiting the phosphorylation of Smad2. Therefore, there is a crosstalk between the non-canonical TGF $\beta /$ ERK1/2 and the canonical TGF $\beta / \mathrm{Smad}$ signaling in LECs EMT.

Evidence suggests that the Notch signaling pathway is a vital regulator in the induction of EMT during embryonic development, cancer metastasis and various fibrotic diseases (21). Activated Jagged/Notch signaling has been confirmed in a large range of fibrotic diseases developed in the kidney, liver and lung (26). Moreover, our former study found that the Notch signaling pathway is upregulated via canonical TGF $32 /$ Smad signaling in LECs EMT, while blockade of the Notch
A

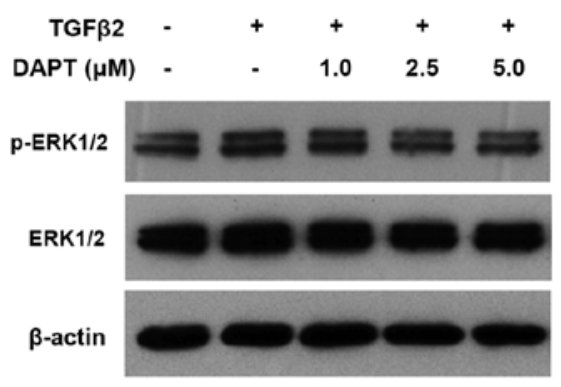

B

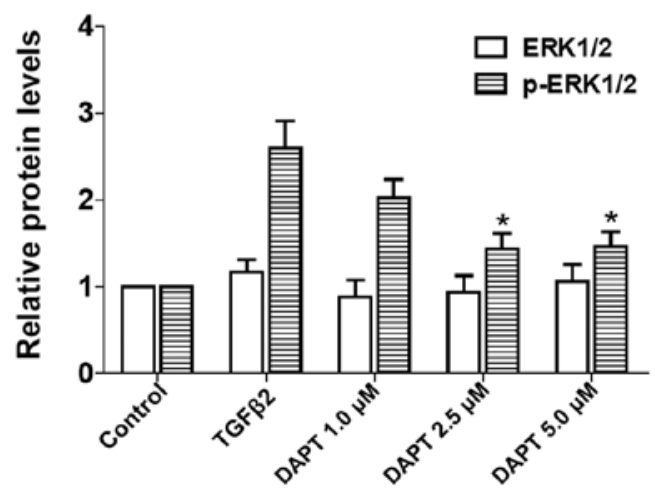

Figure 6. Blockade of Notch pathway by DAPT inhibits the transforming growth factor $\beta$ (TGF $\beta$ )2-induced activation of extracellular signal-regulated kinase (ERK)1/2 pathway. (A) Lens epithelial cells (LECs) were cultured in the absence or presence of TGF 32 with DAPT $(1.0,2.5$ or $5.0 \mu \mathrm{M})$ or DMSO for $30 \mathrm{~min}$, while the expression levels of p-ERK1/2 and ERK1/2 were determined by western blot analysis. (B) Quantification of protein levels from three independent experiments. ${ }^{*} \mathrm{P}<0.05$ vs. TGF $\beta 2$ treated with the DMSO group.

pathway with DAPT markedly reverses TGF $\beta 2$-induced EMT. In this study, we have shown that U0126 attenuates the TGF 32 induced upregulation of Jagged-1, Notch-1 and Notch-2, as well as TGF 22 -induced Notch target genes Hes-1 and Hey-1 expression. These results suggest that non-canonical ERK1/2 signaling also contributes to the TGF 32 -induced activation of the Notch pathway in LECs. Inactivation of ERK1/2 with U0126 abrogates TGF 32 -induced EMT partly by suppressing the Jagged/Notch pathway. Furthermore, we observed that blockade of the Notch pathway by DAPT inhibits the TGFß2-induced activation of the ERK1/2 pathway. This means non-canonical TGF $\beta /$ ERK1/2 signaling can be mediated by the Notch pathway inversely in LECs. Collectively, these data indicate that there is a crosstalk between the ERK1/2 signaling and the Notch pathway in LECs EMT.

In summary, our results provide evidence that the TGF $\beta 2$-induced activation of ERK1/2 is independent of canonical TGF $\beta /$ Smad signaling in human LECs. Inactivation of ERK1/2 signaling with U0126 completely inhibits TGF $\beta 2$-induced EMT in LECs. In addition, the blockade of ERK1/2 signaling inhibits the canonical Smad signaling pathway, as well as the Jagged/Notch pathway. We also found that non-canonical TGF $\beta /$ ERK1/2 signaling can be mediated by the Notch pathway conversely. Thus, findings of this study suggest that ERK1/2 signaling cross-interacts with the canonical TGF $/$ Smad and the Jagged/Notch signaling path- 
ways, thus mediating EMT in LECs. Therefore, ERK inhibitor may have therapeutic value in the prevention and treatment of ASC and PCO.

\section{Acknowledgements}

We would like to thank Professor Fu Shang for kindly providing the SRA01/04 human LEC line for this study. The study was funded by the grant from the Guangdong Natural Science Foundation (S2012020010878).

\section{References}

1. McCarty CA and Taylor HR: Recent developments in vision research: light damage in cataract. Invest Ophthalmol Vis Sci 37: 1720-1723, 1996

2. Nathu Z, Dwivedi DJ, Reddan JR, Sheardown H, Margetts PJ and West-Mays JA: Temporal changes in MMP mRNA expression in the lens epithelium during anterior subcapsular cataract formation. Exp Eye Res 88: 323-330, 2009.

3. Shin EH, Basson MA, Robinson ML, McAvoy JW and Lovicu FJ: Sprouty is a negative regulator of transforming growth factor $\beta$-induced epithelial-to-mesenchymal transition and cataract. Mol Med 18: 861-873, 2012.

4. Apple DJ, Solomon KD, Tetz MR, et al: Posterior capsule opacification. Surv Ophthalmol 37: 73-116, 1992.

5. Hodge WG: Posterior capsule opacification after cataract surgery. Ophthalmology 105: 943-944, 1998.

6. Srinivasan Y, Lovicu FJ and Overbeek PA: Lens-specific expression of transforming growth factor betal in transgenic mice causes anterior subcapsular cataracts. J Clin Invest 101: 625-634, 1998.

7. de Iongh RU, Wederell E, Lovicu FJ and McAvoy JW: Transforming growth factor-beta-induced epithelial-mesenchymal transition in the lens: a model for cataract formation. Cells Tissues Organs 179: 43-55, 2005.

8. Wallentin N, Wickström K and Lundberg C: Effect of cataract surgery on aqueous TGF-beta and lens epithelial cell proliferation. Invest Ophthalmol Vis Sci 39: 1410-1418, 1998.

9. Meacock WR, Spalton DJ and Stanford MR: Role of cytokines in the pathogenesis of posterior capsule opacification. Br J Ophthalmol 84: 332-336, 2000.

10. Awasthi N, Guo S and Wagner BJ: Posterior capsular opacification: a problem reduced but not yet eradicated. Arch Ophthalmol 127: 555-562, 2009.

11. Allen JB, Davidson MG, Nasisse MP, Fleisher LN and McGahan MC: The lens influences aqueous humor levels of transforming growth factor-beta 2. Graefes Arch Clin Exp Ophthalmol 236: 305-311, 1998.
12. Eldred JA, Dawes LJ and Wormstone IM: The lens as a model for fibrotic disease. Philos Trans R Soc Lond B Biol Sci 366: 1301-1319, 2011.

13. Akhurst RJ and Hata A: Targeting the TGF $\beta$ signalling pathway in disease. Nat Rev Drug Discov 11: 790-811, 2012.

14. Li J, Tang X and Chen X: Comparative effects of TGF- $\beta 2 / \mathrm{Smad} 2$ and TGF- $\beta 2 / \mathrm{Smad} 3$ signaling pathways on proliferation, migration, and extracellular matrix production in a human lens cell line. Exp Eye Res 92: 173-179, 2011.

15. Dawes LJ, Sleeman MA, Anderson IK, Reddan JR and Wormstone IM: TGFbeta/Smad4-dependent and -independent regulation of human lens epithelial cells. Invest Ophthalmol Vis Sci 50: 5318-5327, 2009.

16. Chung EJ, Chun JN, Jung SA, Cho JW and Lee JH: TGF- $\beta$-stimulated aberrant expression of class III $\beta$-tubulin via the ERK signaling pathway in cultured retinal pigment epithelial cells. Biochem Biophys Res Commun 415: 367-372, 2011.

17. Chen XF, Zhang HJ, Wang HB, et al: Transforming growth factor- $\beta 1$ induces epithelial-to-mesenchymal transition in human lung cancer cells via PI3K/Akt and MEK/Erk1/2 signaling pathways. Mol Biol Rep 39: 3549-3556, 2012.

18. Aomatsu K, Arao T, Sugioka K, et al: TGF- $\beta$ induces sustained upregulation of SNAI1 and SNAI2 through Smad and non-Smad pathways in a human corneal epithelial cell line. Invest Ophthalmol Vis Sci 52: 2437-2443, 2011.

19. Choi J, Park SY and Joo CK: Transforming growth factorbeta1 represses E-cadherin production via slug expression in lens epithelial cells. Invest Ophthalmol Vis Sci 48: 2708-2718, 2007.

20. Xie L,Law BK, Chytil AM, Brown KA, Aakre ME and Moses HL: Activation of the Erk pathway is required for TGF-betal-induced EMT in vitro. Neoplasia 6: 603-610, 2004.

21. Wang Z, Li Y, Kong D and Sarkar FH: The role of Notch signaling pathway in epithelial-mesenchymal transition (EMT) during development and tumor aggressiveness. Curr Drug Targets 11: 745-751, 2010

22. Neuzillet C, Tijeras-Raballand A, de Mestier L, Cros J, Faivre S and Raymond E: MEK in cancer and cancer therapy. Pharmacol Ther 141: 160-171, 2013

23. Tanahashi T, Osada S, Yamada A, et al: Extracellular signalregulated kinase and Akt activation play a critical role in the process of hepatocyte growth factor-induced epithelial-mesenchymal transition. Int J Oncol 42: 556-564, 2013.

24. Pacheco-Domínguez RL, Palma-Nicolas JP, López E and López-Colomé AM: The activation of MEK-ERK1/2 by glutamate receptor-stimulation is involved in the regulation of RPE proliferation and morphologic transformation. Exp Eye Res 86: 207-219, 2008.

25. Zhang YE: Non-Smad pathways in TGF-beta signaling. Cell Res 19: 128-139, 2009.

26. Leask A: Targeting the jagged/notch pathway: a new treatment for fibrosis? J Cell Commun Signal 4: 197-198, 2010. 